\title{
Dosvox: tecnologia que amplia a comunicação homem-máquina para deficientes visuais
}

\author{
Francisco das Chagas Brito dos Santos ${ }^{1}$, Aline Montenegro Leal Silva ${ }^{1}$, Francisco \\ Alysson da Silva Sousa ${ }^{1}$, Keylla Maria de Sá Urtiga Aita ${ }^{1}$, Francisco Renato Lima ${ }^{1}$, \\ Vinícius Ponte Machado ${ }^{1}$ \\ ${ }^{1}$ Universidade Federal do Piauí (UFPI) \\ Centro de Educação Aberta e a Distância (CEAD) \\ Curso de Licenciatura em Computação \\ fbrito5063@gmail.com, alineleal@ufpi.edu.br, webalysson@ufpi.edu.br, \\ keyllaurtiga@ufpi.edu.br, fcorenatolima@hotmail.com, vinicius@ufpi.edu.br
}

\begin{abstract}
Technology is increasingly present in our society. Access to information is essential in the digital age, but it is important that such technologies provide easy interactions, capable of being detected and correctly interpreted by people with disabilities. This work aims to address the digital inclusion of visually impaired people in society through the Dosvox software. The methodology used was a literature review with a qualitative approach, which raised the difficulties of the inclusion of the visually impaired in society and the relevance of Assistive Technology (AT) to promote this inclusion. It could be concluded that this AT promotes digital accessibility by allowing the visually impaired to work autonomously on the computer or smartphone.
\end{abstract}

Resumo: A tecnologia está cada vez mais presente em nossa sociedade. O acesso às informações é indispensável na era digital, porém é importante que tais tecnologias forneçam interações fáceis, capazes de serem detectadas e corretamente interpretadas por pessoas com deficiência. O presente trabalho objetiva abordar a inclusão digital de deficientes visuais na sociedade por meio do software Dosvox. A metodologia utilizada foi uma revisão bibliográfica de abordagem qualitativa, onde se levantou os percalços da inclusão do deficiente visual na sociedade e a relevância da Tecnologia Assistiva (TA) para promover essa inclusão. Pôde-se concluir que essa TA promove a acessibilidade digital ao permitir que o deficiente visual trabalhe de forma autônoma no computador ou smartphone.

\section{Introdução}

As inovações tecnológicas e o crescimento da área de informática exercem grande impacto nos diversos setores da sociedade. No campo educacional, o uso de softwares específicos para portadores de necessidades especiais facilita o aprendizado e possibilita a inclusão, como é o caso dos deficientes visuais.

Neste sentido, Nascimento (2015) constatou que a dificuldade de se encontrar softwares que atendam às necessidades especiais de certo tipo de usuário é cada vez mais difícil. Em muitos casos os mesmos não são de domínio público, o que dificulta um pouco o acesso pelos deficientes visuais.

Quando o foco são os sistemas computacionais, depara-se com a mesma dificuldade, já que surgem novas tecnologias de hardwares que se utilizam de computadores com funcionalidades cada vez mais complexas, capazes de solucionar as mais amplas variedades de problemas, o que gera uma melhoria na qualidade de vida dos seres humanos [Barbosa 2009].

Ainda segundo Barbosa (2009), a internet surge como uma importante ferramenta de apoio e incentivo para que as pessoas com deficiência possam alcançar uma certa 
independência, através desta rede e o deficiente pode interagir com pessoas, estudar, ter acesso a novos conhecimentos e dispor de recursos que melhorem o seu dia a dia.

Quando se fala deficientes visuais, estes se utilizam de programas com síntese de voz que convertem as informações textuais em áudio, permitindo assim o acesso à web, interagindo através do teclado. Os ampliadores e leitores de tela, os sintetizadores de voz, o sistema braile e o sistema DOSVOX são alguns dos recursos que podem ser citados como ferramentas que atendem as pessoas com deficiência visual [Borges 2009].

Dosvox é um sistema que realiza a comunicação com o usuário deficiente visual através de sintetizadores de voz. A partir de programas específicos e interfaces adaptativas, o software permite a realização de atividades cotidianas possibilitando a aprendizagem, a independência, a capacitação para o trabalho e promovendo o acesso digital às pessoas com deficiência visual [Dosvox 1993].

Duque e Valente (2015) ressaltaram que, no âmbito da internet, falar de acessibilidade significa oferecer condições para que todos possam aproveitar as oportunidades da grande rede. A acessibilidade atribui igual importância a pessoas com ou sem limitações na capacidade de movimento, de percepção, de cognição e de aprendizado. Promover a acessibilidade significa permitir que mais pessoas possam perceber, compreender e utilizar o sistema para usufruir do apoio computacional oferecido por ele.

Sem uma tecnologia de acesso adequada, os deficientes visuais ficam inibidos ou até mesmo impossibilitados de utilizarem plenamente as potencialidades da internet. Norman (2019) afirmou que para isso, deve-se conhecer os usuários finais, saber como realizam suas tarefas, os tipos de imposições e limites que estão sujeitos. Como as interfaces gráficas constituem uma barreira ao acesso de deficientes visuais, estes, para interagir com os sistemas, usam tecnologias de apoio capaz de captar as interfaces e torná-las acessíveis.

Nesse contexto, o presente trabalho tem como objetivo apresentar o árduo caminho da inclusão do deficiente visual, bem como a relevância do sistema Dosvox na conquista dessa inclusão e seu uso por pessoas deficientes visuais. Especificamente: conhecer as principais características e vantagens da utilização do sistema Dosvox para deficientes visuais; pesquisar os ganhos em acessibilidade, tanto em relação ao mundo digital, como ao mundo estudantil e profissional, os quais foram conseguidos com a implantação do sistema Dosvox no Brasil; conhecer as dificuldades dos deficientes visuais no manejo do software; conhecer o que diz a literatura sobre sua acessibilidade e usabilidade.

Sua relevância reside na possibilidade de se chamar a atenção da comunidade escolar e das autoridades competentes para a importância da acessibilidade da pessoa portadora de deficiência visual, seus potenciais reprimidos, e a importância da adaptação dos ambientes escolares e trabalhistas, para que estas pessoas possam exercer a sua cidadania.

\section{Deficientes visuais e as dificuldades da inclusão}

Aranha (2004) destacou que de acordo com o Instituto Brasileiro de Geografia e Estatística (IBGE), 24\% dos Brasileiros são portadores de algum tipo de deficiência, sofrem discriminação de algum tipo em suas comunidades, sendo excluídos de ingressarem no mercado de trabalho. Essa exclusão social de pessoas portadoras de deficiência ou que possuem alguma necessidade especial é tão antiga quanto a própria história da socialização humana.

Ainda de acordo com Aranha (2004), a natureza das sociedades, desde os mais remotos tempos, sempre foram discriminantes e inabilitavam as pessoas portadoras de deficiências, marginalizando-as e consequentemente as privava do uso de sua liberdade. Essa parcela da população, desrespeitada, sem direito a atendimento, tolhida de seus direitos, era alvo das mais 
diversas formas de preconceitos e discriminação. E inerente ao homem, é bem mais fácil voltar a sua atenção às limitações e impedimentos, do que prestar atenção às capacidades e potenciais que estas pessoas possuem.

Santos et al. (2017) ressaltaram que a inclusão social oferece oportunidades a todas as pessoas de terem acesso às Tecnologias de Informação e Comunicação (TICs) - segundo os autores a ideia seria que todas as pessoas tivessem acesso às informações, principalmente a população de baixa renda, para que pudessem fazer pesquisas, mandar e-mails, se comunicar, enfim, que pudessem facilitar as suas vidas através das tecnologias assistivas. Segundo os autores, a inclusão social, que ocorre através da escola, deveria explorar as possibilidades em que a tecnologia assistiva no mundo digital pode ajudar os alunos com deficiências visuais a interagir com as disciplinas que estão sendo aplicadas em sala de aula. Antes das tecnologias assistivas, estes alunos não tinham acesso a oportunidades, ao desenvolvimento acadêmico e social, ficando assim excluídos.

Ainda segundo Santos et al. (2017), a tecnologia assistiva por si só não fará nenhum efeito milagroso, é preciso a formação e existência de profissionais aptos para a sua aplicabilidade e locais preparados para recepcionar os deficientes visuais em relação ao uso de alguns aplicativos.

Segundo Silva, Mól e Santana (2019), é na escola que nossas crianças adquirem conhecimentos que levarão para a vida toda. A escola ajuda a construir a consciência crítica, o raciocínio, o respeito pelas diversidades, e principalmente o convívio social, sendo estes os princípios básicos da escola. Não se pode admitir uma escola que exclua, é preciso políticas públicas em prol de uma escola cada vez mais inclusiva onde se quebre essa visão caótica de uma escola excludente, que isso fique no passado, que se mude no presente para construir um futuro melhor para nossas crianças, futuros cidadãos.

\subsection{Dosvox}

Nos últimos anos, o Núcleo de Computação Eletrônica da Universidade Federal do Rio de Janeiro (UFRJ) tem se dedicado à criação de um sistema de computação voltado a permitir que pessoas com deficiência visual utilizem um computador comum (PC) adquirindo assim, um nível de independência nos estudos e no trabalho [Dosvox 1993].

O Dosvox nada mais é que um sistema desenvolvido para microcomputadores da linha PC, que utiliza a síntese de voz para se comunicar com o usuário deficiente visual, permitindo que esses utilizem o computador, adquirindo assim um alto nível de independência nos estudos e no trabalho. O que diferencia o Dosvox de outros sistemas similares, é que no Dosvox, a comunicação entre humano-computador é muito simples, levando em conta as limitações e especificidades do usuário. O Dosvox estabelece um diálogo amigável com o usuário, porque não apenas ler o que está escrito na tela, mas se utiliza de interfaces adaptativas e programas específicos, o que o torna insuperável em qualidade e facilidade de uso por deficientes visuais. Grande parte das mensagens emitidas são feitas em voz humana, com isso ele gera um baixo nível de estresse para o usuário, mesmo em um uso prolongado [Dosvox 1993].

Para Ventavoli (2012), a informática é a principal aliada do ensino, uma vez que se torna atrativa e altamente expressiva quando se fala de educação especial. Destaca ainda que a tecnologia coligada à educação traz grandes benefícios ao aluno deficiente visual, pois estimula seus potenciais e melhora suas habilidades.

Já Barbosa, Martins e Santos (2015) relataram em seu trabalho com alunos deficientes visuais, que o uso das Tecnologias Assistivas promove uma mudança de perspectivas sobre a importância do uso do computador para o deficiente visual, onde por meio de relatos dos próprios alunos eles poderão pesquisar informações, estudar de forma independente e acessar as redes sociais que eles só ouviam falar. 


\subsection{Principais funcionalidades do Dosvox}

Silva (2017) destacou, como função principal do Dosvox, o conhecer do teclado. Segundo o autor, a pessoa cega deve antes de tudo aprender a localizar as teclas do teclado. Seria como um curso de datilografia para os cegos, sendo esta uma das funções básicas do Dosvox, necessária para que a pessoa possa não apenas conhecer o teclado básico, mas sim as demais funções do teclado. Como existe uma variedade muito de grande de configurações de teclado, essa se configura como a etapa inicial e essencial do Dosvox.

Ainda segundo Silva (2017), uma vez que a pessoa cega passa a dominar o teclado, poderá então começar a utilizar as demais funcionalidades disponíveis no sistema, sendo um dos objetivos principais de quem utiliza o Dosvox, a possibilidade de poder escrever um texto e depois poder lê-lo novamente. Para isso poderá utilizar o Edivox, editor de texto muito simples de ser utilizado disponível na opção "Editar texto". Com ele o usuário cria um texto novo, ou pode editar um já existente, lembrando sempre que cada tecla digitada é falada. $\mathrm{O}$ Edivox conta com um corretor ortográfico que ajuda na ora de verificar a ortografia da palavra digitada.

Borges (2015), relata que quando foi lançado o sistema Dosvox, este contava apenas com cinco a seis programas, porém havia um interesse muito grande pela questão dos jogos, mesmo tendo muitos programas disponíveis aos usuários que sentiam a necessidade de elementos lúdicos disponíveis no sistema. Hoje o Dosvox conta com vários jogos divididos em várias categorias dependendo da função a que o jogo se destina. Esta é uma das principais funcionalidades do sistema para a iniciação das crianças deficientes visuais ao mundo da tecnologia, de forma simples e divertida.

Outra opção muito importante, é o leitor de documentos acessado através da opção "Ler Texto", permitindo assim a leitura de textos salvos no computador. Dessa forma, a leitura pode ser iniciada, pausada. Pesquisar strings, é mais uma forma em que a o deficiente visual tem para ter acesso à informação [Silva 2017].

Verifica-se atualmente que as demandas das pessoas deficientes visuais vêm se modificando, e, cada vez mais elas buscam acesso à internet. Para possibilitar esse acesso, o sistema Dosvox disponibiliza a opção "Acesso à rede e internet", permitindo desta forma que a pessoa com deficiência visual possa acessar sites de informações, vídeos, suas caixas de email e muito mais, quebrando de vez com as barreiras da desinformação [Borges 2015].

A princípio o Dosvox parece ser um sistema operacional independente, porém a pessoa deficiente visual poderá acessar os recursos de seu computador fora do Sistema Dosvox. O programa Monitvox que vem instalado junto com o Dosvox, permite a leitura da tela do Windows, permitindo assim a acessibilidade para este ambiente, o que possibilita um primeiro contato do deficiente visual com os leitores de tela.

\section{Metodologia}

O estudo abordado tratou-se de uma revisão da literatura e para tanto, efetuou-se a pesquisa na base de dados da SCIELO (http://www.scielo.br/), na base de dados do Google acadêmico (https://scholar.google.com.br), no site do Congresso Brasileiro de Informática e Educação "cbie.ceie-br.org/2020/” e no site do próprio Dosvox (http://intervox.nce.ufrj.br/dosvox/), utilizando-se de descritores como "Dosvox", "Inclusão", "deficiente visual", e como string principal de pesquisa "Dosvox e a Inclusão do Deficiente Visual".

Por ser uma ferramenta exclusivamente brasileira, foram selecionados apenas trabalhos escritos na língua portuguesa e que foram publicados nos últimos 5 anos, bem como por meio de um percurso cognitivo onde o próprio autor da pesquisa simulou o usuário, testando a funcionalidade do sistema, de forma que ele pudesse sentir e concluir a relevância do Sistema 
Dosvox. Não houve a finalidade de se esgotar e explorar todo o conhecimento existente sobre o assunto, mas apenas de desenvolver uma reflexão sobre o tema proposto.

Selecionou-se então, 11 trabalhos que satisfizessem os critérios da pesquisa como mostra a Tabela 1 .

Tabela 1. Trabalhos Selecionados na Pesquisa.

\begin{tabular}{|l|l|}
\hline \multicolumn{1}{|c|}{ Autor/Ano } & \multicolumn{1}{c|}{ Título } \\
\hline Silva, Freitas e Araújo (2019) & $\begin{array}{l}\text { Acessibilidade: O uso de tecnologias } \\
\text { assistivas para deficientes visuais. }\end{array}$ \\
\hline Bruno e Nascimento (2018) & $\begin{array}{l}\text { Política de acessibilidade: o que dizem as } \\
\text { pessoas com deficiência visual. }\end{array}$ \\
\hline Santos et al (2017) & $\begin{array}{l}\text { Tecnologia Assistiva: um estudo sobre o uso } \\
\text { de aplicativos para deficientes visuais. }\end{array}$ \\
\hline Barbosa, Martins e Santos (2015) & $\begin{array}{l}\text { Uma Experiência no Ensino de Informática } \\
\text { Para Deficientes Visuais no Município de } \\
\text { Guarulhos - PE. }\end{array}$ \\
\hline Silva (2015) & $\begin{array}{l}\text { Análise sobre a usabilidade do Dosvox. } \\
\text { Santinello, Evaristo e Pizzol (2020) }\end{array}$ \\
\hline Duque \& Valente (2015) & $\begin{array}{l}\text { Percepção de um professor cego sobre a } \\
\text { tecnologia assistiva Dosvox para o ensino }\end{array}$ \\
\hline Silva, Mól e Santana (2019) & $\begin{array}{l}\text { Avaliação da acessibilidade e usabilidade do } \\
\text { sistema Dosvox }\end{array}$ \\
\hline Del Piero e Araújo (2017) & $\begin{array}{l}\text { Os Recursos Tecnológicos e de } \\
\text { Acessibilidade para a Pessoa com } \\
\text { Deficiência Visual }\end{array}$ \\
\hline Silva (2017) & $\begin{array}{l}\text { Dosvox: possibilidades de uso pedagógico no } \\
\text { processo de ensino-aprendizagem. }\end{array}$ \\
\hline Melo, Souza e Lima (2020) & $\begin{array}{l}\text { Jogos digitais adaptados para estudantes com } \\
\text { deficiência visual: estudo das habilidades } \\
\text { cognitivas no Dosvox. }\end{array}$ \\
\hline $\begin{array}{l}\text { A Tecnologia Assistiva e a Inclusão } \\
\text { Educacional de Pessoas com Deficiência: um } \\
\text { mapeamento sistemático da literatura. }\end{array}$ \\
\hline
\end{tabular}

\section{Análise e Discussão dos Dados}

Nesse trabalho de pesquisa elencou-se dois eixos de pesquisa: o primeiro tem a ver com os percalços da inclusão da pessoa deficiente visual e o segundo, a relevância da tecnologia assistiva Dosvox na facilitação desta inclusão. Sendo assim, foi feito uma análise dos dados encontrados na pesquisa separando-se esses eixos.

$1^{\circ}$ Os Percalços da Inclusão.

Silva, Freitas e Araújo (2019) em sua pesquisa ressaltaram que as assistências às pessoas portadoras de deficiência visual ainda necessitam evoluir bastante para chegar ao ponto de inclusão na sociedade, pois algumas destas pessoas ainda encontram dificuldades para se inserir de modo independente.

Bruno e Nascimento (2018) demonstraram em seu trabalho quais os desafios para a política pública de educação que são: investimentos em tecnologia assistiva em espaço inclusivo; promover a articulação entre os professores da educação especial e do ensino comum; a oferta dos recursos de TA nas salas de aula, bem como, a formação desses professores para o uso das tecnologias para todas as pessoas com deficiência. 
Já Santos et al. (2017) mostraram que a tecnologia assertiva por si só não fará efeito, precisa-se de profissionais aptos para sua aplicabilidade e locais preparados para recepcionar os deficientes visuais em relação ao uso de alguns aplicativos.

Barbosa, Martins e Santos (2015) relataram, de maneira geral, que o uso das tecnologias assistivas facilitam o acesso da pessoa deficiente visual ao computador, garantindo-lhes maior autonomia por meio da possibilidade de acesso a ambientes virtuais, o que favorece a inclusão social e digital.

\section{$2^{\circ}$ A Relevância da TA Dosvox.}

Silva (2015) destacou a importância do uso do Dosvox por pessoas cegas e de baixa visão, tanto no âmbito social como educacional. Ressaltou ainda, a relevância das políticas sociais e tecnológicas no uso e incentivo de softwares livres, para a promoção da igualdade de condições para esses sujeitos, no que se refere à acessibilidade digital.

Santinello, Evaristo e Pizzol (2020), em seu trabalho "Percepção de um professor cego sobre a tecnologia assistiva Dosvox para o ensino", destacaram que segundo o professor, a TA Dosvox carece de maiores reformulações, principalmente no que tange aos jogos digitais, os quais são muito utilizados no processo inicial de alfabetização das crianças que ele presta atendimento. Porém, o professor reforça que para inicialização do deficiente visual ao mundo digital, a ferramenta Dosvox ainda é o melhor caminho.

Duque e Valente (2015), destacaram que a sua avaliação ficou restrita a apenas um portal e uma avaliadora. Sugerem que seu artigo seja apenas o primeiro de uma série de projetos que visem estudar o sistema Dosvox e assim poder apresentar resultados mais concretos e abrangentes com relação a sua usabilidade e acessibilidade, porem em um estudo preliminar, o Dosvox se mostrou de fácil manuseio.

Silva, Mól e Santana (2019), em seu trabalho, perceberam que os amigos e familiares tem uma enorme contribuição na apresentação dos Recursos Tecnológicos Digitais (RTD) para as pessoas deficientes visuais participantes da pesquisa e constataram que o acompanhamento desse processo também foi uma postura dos familiares e amigos envolvidos. Constataram também que o sistema Dosvox teve lugar de destaque nas indicações dos participantes da pesquisa, por ser o de mais fácil manuseio, ser gratuito, sendo o mais utilizado dentre eles.

Del Piero e Araújo (2017), com base em todo o estudo feito ao longo do seu trabalho, concluíram que o Dosvox é de suma importância dentro dos muros das escolas, no dia a dia do aluno com deficiência visual, principalmente por incentivar o seu uso nos laboratórios de informática como a ferramenta inicial de inserção da pessoa com deficiência visual ao mundo digital.

Silva (2017) ressaltou que os jogos digitais adaptados podem ser uma forma de alcançar a aprendizagem dos conteúdos e o desenvolvimento das habilidades cognitivas, de construir relações sociais, de perceber as atitudes morais e éticas dos jogadores com e sem deficiência visual. As possibilidades da utilização do jogo digital adaptado são inúmeras. A resistência em relação a sua utilização vem, muitas vezes, do professor que não consegue perceber os seus benefícios, ou que não está capacitado para utilizá-los. O Dosvox é uma das principais ferramentas que serve como porta inicial neste processo.

Melo, Souza e Lima (2020), com base em um mapeamento da Literatura, apresentaram como principal contribuição do seu trabalho, uma visão geral da importância dos recursos e serviços de TA desenvolvidos para auxiliar na inclusão, não apenas educacional, da pessoa com deficiência. Neste sentido, seus resultados são uteis tanto para os educadores quanto para as pessoas com deficiência que necessitam fazer uso de tais recursos, como também aos 
pesquisadores que desejam desenvolver outros tipos de tecnologias no âmbito educacional onde ainda há uma grande escassez de soluções.

\section{Conclusão}

Por meio da pesquisa bibliográfica desenvolvida pôde-se perceber a relevância do software Dosvox para inclusão digital de deficientes visuais na sociedade. A metodologia utilizada foi uma revisão bibliográfica de abordagem qualitativa, onde se levantou os percalços da inclusão do deficiente visual na sociedade e a importância da Tecnologia Assistiva para promover essa inclusão.

O sistema Braille abre espaço para estas novas ferramentas, que tornam a comunicação professor aluno bem mais fácil, além de sua simplicidade, custo e de se adequar à realidade dos deficientes visuais. Isto porque as tecnologias assistivas são a ponte que o Braille não oferecia para a comunicação do deficiente visual com aqueles que não possuem essa deficiência. $\mathrm{Na}$ maioria dos casos, nem os professores, nem os familiares sabiam ler o que os cegos escreviam, limitando-se assim a sua comunicação apenas com outros deficientes visuais.

Desta forma, a TA Dosvox quebrou as barreiras de comunicação que existiam entre o professor e aluno deficiente visual, e por que não dizer, do deficiente visual com o mundo que o cerca, pois, a ferramenta não apenas possibilita ao deficiente visual desenvolver a leitura e a escrita, mas acima de tudo, permite o acesso ao mundo digital, rompendo enfim barreiras centenárias da exclusão.

Portanto, foi possível concluir por meio da pesquisa e das informações levantadas, que mesmo apresentando algumas limitações, o sistema Dosvox se apresenta como uma excelente ferramenta de inclusão social do deficiente visual, não apenas no meio estudantil, mas também ao meio social e profissional, ao tempo que facilita a sua comunicação, tornando-os pessoas mais produtivas e indivíduos mais integrados à sociedade. Porém, se não houver compromisso por parte dos órgãos governamentais e colaboração dos segmentos organizados da sociedade, fica praticamente inviável qualquer projeto que vise a inclusão.

\section{Referências}

Aranha, M. S. F. (2004). Educação Inclusiva: transformação social ou retórica, 2004. In: OMOTE, S. (org.). Inclusão: intenção e realidade (pp. 37-60). Marília: Fundepe.

Barbosa, A. F; Martins, R. O; Santos, H. R. M. (2015). Uma Experiência no Ensino de Informática Para Deficientes Visuais no Município de Guarulhos - PE. Disponível em: https://br-ie.org/pub/index.php/wie/article/view/2626/2280, acesso em 20 de novembro de 2020.

Barbosa, S. D. J; Silva, B. S. (2009). Interação Humano-Computador, Rio de Janeiro: Editora Campus-Elzevier, 397p.

Borges, J. A. (2015). Do Braille ao Dosvox - diferença na vida dos cegos brasileiros. Tese Doutorado em Ciências em Engenharia de Sistemas e Computação, Programa de Pósgraduação de Engenharia, Universidade Federal do Rio de Janeiro, RJ. Rio de Janeiro.

Bruno, M. M. G; Nascimento, R. A. L. (2018). Política de Acessibilidade: o que dizem as pessoas com deficiência visual, 2018. Disponível em: https://www.scielo.br/scielo.php?script=sci_abstract\&pid=S217562362019000100206\&lng=en\&nrm=iso/\&tlng=pt, acesso em 20 de nov. 2020. 
Del Piero, A. B. P; Araújo, I. M. (2017). DOSVOX: Possibilidade de uso pedagógico no processo ensino-aprendizagem. Universidade Federal Rural da Amazônia - UFRA, Brasil.

Dosvox. O que é o Dosvox. (1993). Disponível em: $<$ http://intervox.nce.ufrj.br/dosvox/intro.htm>. Acesso em: 11 jul. 2020.

Duque, J. P; Valente, W. A. G. (2015). Avaliação da acessibilidade e usabilidade do sistema DOSVOX. Centro de Ensino Superior de Juiz de Fora - MG - Brasil.

Melo, A. C. de M; Souza, E. P. R; Lima, J. V (2020). A Tecnologia Assistiva e a Inclusão Educacional de Pessoas com Deficiência: um mapeamento sistemático da literatura. Em, IX Congresso Brasileiro de Informática na Educação (CBIE 2020) Anais do XXXI Simpósio Brasileiro de Informática na Educação (SBIE 2020).

Nascimento, R. A. L. do. (2015). O Impacto dos Recursos de Tecnologia Assistiva na Educação e Inclusão da Pessoa com Deficiência Visual. 2015. 131 f. Dissertação (Mestrado em Educação) - Universidade Federal da Grande Dourados, Dourados.

Norman, D. A. (2019). The Invisible Computer: why good products can fail, the personal computer is so complex and information appliances are the solution. The MIT Press.

Santinello, J.; Evaristo, E. de F; Pizzol, A. D. (2020). Percepção de Um Professor Cego Sobre a Tecnologia Assistiva Dosvox Para o Ensino. Disponível em: https://scholar.google.com.br/scholar?hl=pt-

BR\&as sdt $=0 \% 2 \mathrm{C} 5 \& \mathrm{q}=\mathrm{PERCEP} \% \mathrm{C} 3 \% 87 \% \mathrm{C} 3 \% 83 \mathrm{O}+\mathrm{DE}+\mathrm{UM}+\mathrm{PROFESSOR}+\mathrm{CEGO}+$ SOBRE+A+TECNOLOGIA+ASSISTIVA+DOSVOX+PARA+O+ENSINO\&btnG $=$ acesso em 20 de nov. 2020.

Santos, J. P., de Oliveira, L. C., Salgado, M. H. V., \& da Silva, P. P. (2017). TECNOLOGIA ASSISTIVA: um estudo sobre o uso de aplicativos para deficientes visuais. Brasil Para Todos-Revista Internacional, 4(1), 59-69.

Silva, D. P. R.; Freitas, E. V.; Araújo, L. S. (2019). ACESSIBILIDADE, o uso de tecnologias assistivas para deficientes visuais. em:https://scholar.google.com.br/scholar?hl=pt-

$\mathrm{R} \&$ as_sdt $=0 \% 2 \mathrm{C} 5 \& \mathrm{q}=\mathrm{ACESSIBILIDADE}+\mathrm{O}+$ uso + de + tecnologias + assistivas + para + defic ientes+visuais\&btnG $=$ acesso em 20 de Novembro de 2020.

Silva, K.A.D. (2015). Análise Sobre a Usabilidade do Dosvox. In Anais do Congresso Nacional Universidade EAD Software Livre (Vol. 1, No. 6).

Silva, W. P. (2017). Jogos digitais adaptados para estudantes com deficiência visual: estudo das habilidades cognitivas no Dosvox, 2017 disponível em: https://scholar.google.com.br/citations? user $=0 \mathrm{uFJN20AAAAJ} \& h l=$ pt-BR acesso em 20 nov.2020.

Silva, W. P., Mól, G. de S; Santana, R. de O. (2019). Os Recursos Tecnológicos e de Acessibilidade para a Pessoa com Deficiência Visual. Disponível em: https://www.scielo.br/scielo.php?script=sci_abstract\&pid=S217562362019000100206\&lng=en\&nrm=iso/\&tlng=pt, acesso em 20 de nov. 2020.

Ventavoli. (2012). A informática como ferramenta e proposta educativa aos indivíduos portadores de Deficiência Visual, 2012. Mococa. 